\title{
Bankenarchitekturen im Informationszeitalter - Zur Rolle des Geschäftsmodells
}

Bernd Heinrich, Susanne Leist

1 Problemstellung

2 Geschäftsnetzwerke der Banken

2.1 Potenziale der Produktion von Bankdienstleistungen in Unternehmensnetzwerken

2.2 Vision des Bankgeschäfts im Informationszeitalter

2.3 Auswirkungen auf das Bankgeschäft

3 Kompetenzzentrum Bankenarchitekturen im Informationszeitalter

3.1 Gestaltungsebenen einer Bankenarchitektur

3.2 Bankenarchitekturen als Grundlage für das Geschäftsnetzwerk

3.3 Zielsetzung des Kompetenzzentrums

3.4 Vorgehensweise und Untersuchungsmethodik des Kompetenzzentrums

3.5 Fokussierung auf die Rolle des Service Integrators

4 Das Geschäftsmodell als strategische Ebene der Bankenarchitektur

4.1 Positionierung in Unternehmensnetzwerken auf der Grundlage von Geschäftsmodellen

4.2 Fokussierung des Geschäftsmodells für die Rolle des Service Integrators

5 Zusammenfassung

6 Literatur 


\section{Problemstellung}

Deregulierte Märkte, anspruchsvollere Kunden, variantenreiche Produkte, Eindringen neuer Wettbewerber, Zusammenwachsen von Bank- und Versicherungsleistungen und vieles mehr sind Zeichen eines nachhaltigen und tiefgreifenden Strukturwandels auf dem Bankenmarkt, der sich insbesondere im Retailbereich mit enorm hoher Geschwindigkeit vollzieht.

Ursachen auf nationaler Ebene sind dafür einerseits die zunehmende Wettbewerbsdynamik, die sich aufgrund der gesättigten Inlandsmärkte, neuer Entwicklungen der Informations- und Kommunikationstechnologien und des branchenübergreifenden Wettbewerbs (Allfinanzkonzept) ergibt, sowie andererseits die Verschiebung der Kundenbedürfnisse [Choi 1997, S. 1]. Der sogenannte Wertewandel beim Kunden stellt dabei eine besondere Herausforderung für die Banken dar. Während die Ansprüche des Kunden bezüglich der (Beratungs-) Qualität sowie der Flexibilität und Verfügbarkeit des Dienstleistungsangebotes stetig steigen, sinkt gleichzeitig die Loyalität der Kunden gegenüber der eigenen Bank. Letzteres wird durch den Aspekt verstärkt, dass ein Wechsel der Bankverbindung durch die Inanspruchnahme beispielsweise von Direktbanken für den Kunden deutlich einfacher geworden und meist mit weniger Kosten verbunden ist.

Die Strukturänderungen ergeben sich darüber hinaus auch durch den Wandlungsprozess im internationalen Kontext [Choi 1997, S. 1]. Zentral für diese Entwicklungen ist der Trend zur Globalisierung. Hierzu haben Liberalisierungsbemühungen der einzelnen Länder bezüglich eines freien Kapitalverkehrs und Marktzugangs, Entwicklungen der Informations- und Kommunikationstechnologien sowie die Diffusion innovativer Finanzprodukte wesentlich beigetragen [Choi 1997, S. 2].

Mehr denn je ist daher eine konsequente Ausrichtung auf Marktanforderungen, Kundenbedürfnisse und Kernkompetenzen notwendig. Vor diesem Hintergrund beschäftigt sich der vorliegende Beitrag mit der Entwicklung einer Architekturgrundlage, die eine den Anforderungen entsprechende flexible Abwicklung des Bankgeschäfts ermöglicht. Aufbauend auf den Potenzialen der Unternehmensnetzwerke im Bankensektor werden im anschließenden Abschnitt 2 eine Vision und Entwicklungstendenzen des Bankgeschäfts im Informationszeitalter vorgestellt. Der nachfolgende Abschnitt 3 stellt das Kompetenzzentrum Bankenarchitekturen im Informationszeitalter vor, das sich mit der Fragestellung beschäftigt, welche Geschäfts-, Prozess- und Informationssystemarchitektur den Banken zugrunde liegen soll, die in einem Netzwerk kooperieren. Erste Ergebnisse des Kompetenzzentrums, die sich auf die Ebene des Geschäftsmodells der Bankenarchitektur beziehen, werden in Abschnitt 4 zusammengefasst. Den Abschluss bildet der Abschnitt 5 mit einer Zusammenstellung der wichtigsten Ergebnisse. 


\section{Geschäftsnetzwerke der Banken}

\subsection{Potenziale der Produktion von Bankdienstleistungen in Unternehmensnetzwerken}

Ein Unternehmensnetzwerk stellt eine ,,auf die Realisierung von Wettbewerbsvorteilen zielende Organisationsform ökonomischer Aktivitäten dar, die sich durch komplex-reziproke, eher kooperative denn kompetitive und relativ stabile Beziehungen zwischen rechtlich selbständigen, wirtschaftlich jedoch zumeist abhängigen Unternehmungen [auszeichnet]" [Sydow 1992, S. 82]. Charakteristisches Merkmal von Unternehmensnetzwerken ist somit, dass die beteiligten Partner innerhalb der Wertschöpfungskette jeweils spezielle Teilleistungen erbringen, deren Bündelung schließlich zu einem Endprodukt bzw. einer Dienstleistung führt [Fugmann et al. 1999, S. 240].

Vorteile eines solchen Unternehmensnetzwerkes werden in der Reduktion der unternehmensinternen Komplexität durch eine Fokussierung auf die Kernkompetenzen gesehen. Dabei wird angenommen, dass die Konzentration auf Kernkompetenzen nicht nur aus wirtschaftlichen (Reduktion der Fertigungstiefe), sondern auch aus organisatorischen Erwägungen vorteilhaft ist. So ermöglicht die organisatorische Trennung beispielsweise von Vertrieb und Infrastrukturmanagement, dass die beiden Bereiche gemäß ihren unterschiedlichen Zielsetzungen und Kulturen autonom agieren können [Hagel/Singer 1999, S. 134]. Die wesentliche Aufgabe des Vertriebes besteht dabei in der Identifikation, Gewinnung und Betreuung der Kunden. Wesentliches Charakteristikum ist demnach die Ausrichtung am Kunden, die insbesondere durch dezentrale, möglichst nahe am Kunden positionierte Vertriebseinheiten, hohe Flexibilität und kommunikationsstarke Verkäufer bzw. Kundenbetreuer gekennzeichnet ist. Demgegenüber besteht die Aufgabe des Infrastrukturmanagements in der Sicherstellung einer effizienten, organisatorischen und technischen Geschäftsabwicklung. Charakteristische Merkmale für diesen Bereich sind demnach Ausrichtung an Kosten, weitgehende Standardisierung, Abwicklung hoher Transaktionsvolumina (und damit Realisierung von Skaleneffekten) [Hagel/Singer 1999, S. 135)]. 
Die Gegensätzlichkeit der genannten Beispiele (Vertrieb und Infrastrukturmanagement) trifft in besonderer Weise auch für Banken zu. Die Notwendigkeit der Einbeziehung des externen Faktors bei der Produktion von Bankdienstleistungen verstärkt einerseits die Bedeutung nahe am Kunden positionierter Vertriebsstellen und der Dezentralität des Vertriebes. Andererseits unterliegt die Produktion von Bankdienstleistungen aufgrund ihrer Auftragsgebundenheit in der Regel hohen Schwankungen. So ergeben sich Spitzenzeiten (beispielsweise zu Börsenbeginn), in denen Bankdienstleistungen verstärkt nachgefragt werden. Dies macht die Größe, d.h. in diesem Fall die Fähigkeit, viele Transaktionen kostengünstig und schnell zu verarbeiten, zum Wettbewerbsvorteil.

Die Bandbreite des Produktangebots der Banken wird durch einerseits stark standardisierte (sogenannte „,commodities“) sowie stark spezialisierte Produkte aufgespannt. Während die spezialisierten Produkte dasjenige Differenzierungspotenzial liefern, welches die Finanzdienstleister zur Kundenbindung und damit zur Gewinnerzielung benötigen, bieten die commodities aufgrund ihrer interorganisatorischen Standardisierung keinerlei Differenzierungschancen [Fugmann et al. 1999, S. 245]. Dennoch bestehen für die Produktion stark standardisierter oder spezialisierter Produkte hohe Eintrittsbarrieren, da im ersten Fall der Wettbewerb über den Preis und im zweiten Fall über Know-how entschieden wird. Die Möglichkeit, sich als Bank in einem Bereich zu spezialisieren und dennoch andere Bereiche mit einzubeziehen, ist in einem Netzwerkverbund realisierbar.

Abgesehen vom höheren Koordinationsaufwand eines Unternehmensverbundes spezialisierter Banken (im Vergleich zu einer Universalbank) werden einem Verbund auch deshalb Nachteile zugesprochen, weil die einzelnen spezialisierten Banken die Kundenanforderungen nicht mehr in ihrer gesamten Bandbreite sehen. Der Mangel, Cross-Selling-Potenziale zu erkennen und aus einer Bandbreite von Kundenbedürfnissen Visionen für neue Produkte zu entwickeln, kann allerdings durch die Existenz einer Vertriebsbank kompensiert werden, die den Kunden möglichst umfassend betreut und das Wissen über die Kundenbedürfnisse an die Verbundpartner weitergibt.

\subsection{Vision des Bankgeschäfts im Informationszeitalter}

Zu einem Verbund spezialisierter Unternehmen wurde am Institut für Wirtschaftsinformatik eine Vision erarbeitet, deren Grundlage in verschiedenen Projekten ${ }^{1}$ mit Unternehmen in Europa und den USA weiterentwickelt wurde. Abbildung 1 stellt die für das Bankgeschäft im Informationszeitalter erarbeitete Vision dar.

Erste Grundlagen der Vision entstanden aus Arbeiten des CC Electronic Business Networking (CC eBN, März 1996 bis März 1998) und des CC International Business Networking (CC iBN, März 1998 bis Februar 2000), in denen ein Vorgehens- und Organisationsmodell für die Implementierung multinationaler Geschäftsnetzwerke mit Schwerpunkt auf die Integration der Applikationen (CC eBN) bzw. der Prozesse (CC iBN) entwickelt wurde. Vgl. hierzu u.a. [Fleisch et al. 1999], [Österle et al. 2000]. Die Ausrichtung der Vision für das Bankgeschäft wurde im CC Bankenarchitekturen im Informationszeitalter entwickelt. 




Abbildung 1: Geschäftsnetzwerk der Banken im Informationszeitalter [Leist/Winter 2000b]

In dieser Vision sind zunächst nur die Rollen des Bankgeschäfts abgebildet und nicht ihre Zugehörigkeit zu einem Unternehmen. Es werden grundsätzlich folgende Rollen unterschieden [Leist/Winter 2000a, S. 154-156]:

- Den Ausgangspunkt des Netzwerkes stellt der End Consumer dar. Er kann in dieser Vision Produkte und Leistungen auf zwei Arten beziehen: Entweder bemüht er den Service Integrator, ihm Angebote zur Befriedigung seiner Bedürfnisse zu unterbreiten, oder er stellt sich selbstständig mit Hilfe des Business Bus (wie beispielsweise im Internet) Produkte und Leistungen zusammen, die der Service Integrator anbietet.

- Im Mittelpunkt des Netzwerkes befindet sich der Service Integrator, der Bankprodukte und -leistungen anbietet bzw. zu einer individuellen Lösung für den Kunden bündelt. Voraussetzungen dafür sind natürlich genaue Kenntnisse über die Bedürfnisse der Kunden sowie Fähigkeiten, den Kunden bei der Auswahl der Lösungen gut zu beraten. Dazu zählen im Weiteren Aufgaben wie beispielsweise das Kanalmanagement oder die Überwachung von Risikopositionen. Die Bankprodukte und -leistungen werden nicht vom Service Integrator selbst erstellt, sondern er bezieht sie von sogenannten Shared und Exclusive Service Providern. Dabei liegt es in seiner Verantwortung, wichtige Informationen über die Bedürfnisse der Kunden an die Provider weiterzugeben. 
- Shared Service Provider bieten ihre Dienstleistungen (z.B. die Wertschriftenabrechnung, das Clearing oder den Betrieb des Rechenzentrums) mehreren Service Integratoren an. Für viele von Ihnen ist es sinnvoll, ihre Leistungen zu standardisieren. Da standardisierte Leistungen vergleichbar werden, stehen sie im Wettbewerb zueinander und differenzieren sich durch den Preis. Für sie ist es somit naheliegend, solche Funktionen des Bankgeschäfts zu übernehmen, mit denen hohe Transaktionsvolumina und damit Skaleneffekte erreicht werden können.

- Im Gegensatz dazu bieten Exclusive Service Provider ihre Produkte und Leistungen nur einem einzigen Service Integrator an. Aufgrund der Exklusivität ihrer Produkte und Leistungen sowie der Beziehung zum Service Integrator besteht zwischen den Exclusive Service Providern kein direkter Wettbewerb. Der rekursive Pfeil an den Exclusive Service Providern deutet an, dass bestimmte Provider übergreifend andere Provider koordinieren, wenn sie in einem Netzwerk gemeinsam mit einem Service Integrator kooperieren. Auf diese Weise können Funktionen wie z.B. ein unternehmensweites Controlling oder Rechtswesen dargestellt werden.

- Als Public Services werden beispielsweise Beglaubigungen und Dienste angeboten, für die besondere Anforderungen hinsichtlich ihrer Glaubwürdigkeit oder Zuverlässigkeit bestehen und die deshalb von öffentlichen Organisationseinheiten, wie beispielsweise Aufsichtsbehörden oder Institutionen mit öffentlich-rechtlichem Charakter, bereitgestellt werden.

- Die Beziehungen zwischen Service Integrator und Exclusive Service Provider können aufgrund ihrer Exklusivität individuell festgelegt werden, während bei einem Shared Service Provider konsequenterweise standardisierte Beziehungen genutzt werden. Die Menge aller technischen und organisatorischen Infrastrukturkomponenten, die notwendig sind, um standardisierte Beziehungen aufbauen zu können, wird als Business Bus [Österle 1999] bezeichnet. Der Business Bus stellt somit nicht nur eine einheitliche Infrastruktur zur Verfügung, sondern auch Regeln, die den Austausch von Informationen vereinfachen, wie das in ähnlicher Weise schon heute für eine begrenzte Anzahl von Informationen auch mit S.W.I.F.T. möglich ist (vgl. Abschnitt 3.2).

Die Zuordnung der einzelnen Rollen zu rechtlich und wirtschaftlich autonomen Unternehmen ist in dieser Vision nicht zwingend erforderlich. Entscheidend ist, dass die Rollen von autonomen Unternehmenseinheiten wahrgenommen werden, die nicht mittels zentraler Hierarchie sondern mittels Marktmechanismen koordiniert werden.

Erste Ansätze der Realisierung eines kooperierenden Verbundes weitgehend unabhängiger Unternehmen sind heute schon am Beispiel der Volksbanken und Raiffeisenbanken sichtbar. Charakteristisch für diesen Verbund ist dabei die Aufteilung in Zentral- und Spezialinstitute. Mit dieser Struktur werden wesentliche Vorteile von Unternehmensnetzwerken realisiert. Einerseits verfügt der Verbund über ein sehr breit abdeckendes Filialnetz, das von autonomen Volksbanken und Raiffeisenbanken geführt wird. Dies ermöglicht den einzelnen Banken, die sowohl Aufgaben des Service Integrators wie auch des Exclusive Service Providers wahrnehmen, sehr schnell Veränderungen der Kundenbedürfnisse wahrzunehmen und 
aufgrund ihrer weitgehenden Unabhängigkeit auch flexibel Anpassungsmaßnahmen durchführen zu können. Andererseits werden durch Kooperationen einzelner Banken gemeinschaftlich nutzbare Servicedienstleistungen aufgebaut, die Synergiepotenziale schaffen, die die teilweise sehr kleinen einzelnen Volksbanken und Raiffeisenbanken für sich nicht erreichen würden [vgl. hierzu Fugmann et al. 1999, S. 246-248]. Außerdem gehören dem Verbund zentrale Institute wie die DG-Bank an, die auch aufgrund ihrer Grösse in der Lage sind, beispielsweise im Konsortium große Emissionen zu begleiten. Die Vision des Geschäftsnetzwerks im Informationszeitalter geht aber noch über eine solche Kooperation von Unternehmen in einem Verbund hinaus. So ist durch den Business Bus nicht nur in technischer Hinsicht, sondern auch auf fachlicher Ebene die jederzeitige Kooperation mit Unternehmen gewährleistet, die nicht dem Verbund angehören, wenn diese die Standards des Business Bus annehmen. Darüber hinaus stellt der Verbund einen Spezialfall der Vision dar, da die Kapitalgeber der Banken ihre Kunden sind (Inhaber von Genossenschaftsanteilen) und somit die Banken aus einer Vermischung von Kunden- und Kapitalgeberinteressen geführt werden.

\subsection{Auswirkungen auf das Bankgeschäft}

Der Verbund unabhängiger spezialisierter Banken, die sich auf ihre Kernkompetenzen konzentrieren und in einem Netzwerk kooperieren, ermöglicht jedem Verbundmitglied eine konsequente Weiterentwicklung seiner Fähigkeiten. Beispielsweise könnten Banken, die hohe Transaktionsvolumina im Zahlungsverkehr oder im Bereich der Wertschriftenabwicklung verarbeiten, diese Kompetenz in Zukunft ausbauen und einem größeren Kundenkreis anbieten. Aufgrund des Größenvorteils dieser Rolle würden sicherlich nur wenige, aber sehr große Transaktionsbanken entstehen. Demgegenüber werden sich aufgrund des hohen Bedarfs individueller Leistungen viele Institute auf deren Produktion spezialisieren. Der Integrator, der sich auf die Bedürfnisse des Kunden ausrichtet und dabei die Prozesse des Kunden in den Mittelpunkt stellt, wird in Zukunft nicht ausschließlich Produkte bzw. Produktkombinationen, sondern insbesondere auch Lösungen anbieten. Im Unterschied zu Produktkombinationen orientieren sich Lösungen an Lebensereignissen oder Situationen, in denen der Kunde zuerst Hilfestellung bei der Strukturierung seines Problems und der Spezifizierung des Bedarfs an Produkten benötigt. Dies ist in der Regel z.B. bei der Geburt des ersten Kindes oder beim Bau eines Einfamilienhauses erforderlich (weitere Beispiele hierzu finden sich u.a. im Angebot von Quicken [Quicken 2000] oder Swiss Life [Swiss Life 2000]).

Die Beispiele deuten gleichzeitig eine weitere Entwicklungstendenz an: Da die Kundenbedürfnisse sich nicht nach den Branchenstrukturen richten, sondern in vielen Fällen ganzheitliche Lösungen erfordern, wird ein Unternehmensnetzwerk im Informationszeitalter nicht nur spezialisierte Banken, sondern je nach Ausrichtung auch bankenfremde Unternehmen, wie beispielsweise Versicherungen, Ausbildungsstätten, Architekten oder Bauunternehmen, integrieren.

Da es dem Service Integrator noch am einfachsten im Unternehmensnetzwerk möglich ist, eine breite Sicht auf den Kunden zu erhalten, wird er diese in Form 
von Anforderungen an seine zuliefernden Verbundpartner weiterleiten. Auf diese Weise ist es möglich, den Unternehmensverbund nach den Bedürfnissen des Kunden auszurichten. Somit erhält der Service Integrator die zentrale Rolle der Vision des Bankgeschäfts im Informationszeitalter.

\section{Kompetenzzentrum Bankenarchitekturen im Informationszeitalter}

Die Vision spezialisierter Banken, die in einem Unternehmensverbund kooperieren, lässt offen, welche Maßnahmen in organisatorischer und technischer Hinsicht für Banken zu ergreifen sind, um sich den neuen Anforderungen stellen zu können. So ergeben sich grundsätzliche Fragen, wie beispielsweise [Leist/Winter 2000a, S. 158]:

- Welche Methoden unterstützen eine Bank bei der Suche der adäquaten Rolle im Netzwerk?

- Ist die Anpassung klassischer Prozessmodelle an die neuen Aufgaben im Frontbzw. Back-Office ausreichend oder sind grundsätzlich neue Modelle zu erarbeiten?

- Welche Vereinbarungen sind im Business Bus zu treffen, damit eine Kommunikation bzw. ein Leistungsaustausch zwischen den Verbundpartner möglich ist?

Diese Fragen werden im Kompetenzzentrum Bankenarchitekturen im Informationszeitalter (CC BAI) untersucht. Das CC BAI ist Teil des Forschungsprogramms „Business Engineering HSG“ am Institut für Wirtschaftsinformatik (IWI) der Universität St.Gallen und beschäftigt sich mit Architekturkonzepten vernetzter Banken. In diesem Projekt arbeitet das IWI mit sechs Partnerunternehmen (Credit Suisse, Dresdner Bank, Migrosbank, RBA-Service, Sparkassen Informatik Zentrum und Systor) für einen Zeitraum von zwei Jahren zusammen, um auf der Grundlage von Good Practices Referenzmodelle und Methoden zu entwickeln, die die Gestaltung von Unternehmensnetzwerken im Bankbereich unterstützen [Leist/ Winter 1999a]. Grundlage des Kompetenzzentrums ist eine Bankenarchitektur, die als Gestaltungsebenen Geschäftsmodelle, Prozesse, Applikationen und Softwaremodule umfasst (siehe Abbildung 2) [Leist/Winter 1999b, S. 4-5].

\subsection{Gestaltungsebenen einer Bankenarchitektur}

In diesem Abschnitt wird der Aufbau einer Bankenarchitektur vorgestellt, die sich an den Gestaltungsebenen des Business Engineering orientiert [vgl. u.a. Brenner 1995, Österle 1995]. Mit dem Einsatz des Business Engineering wird das Ziel verfolgt, die Reorganisation und Neuausrichtung des Unternehmens methodisch zu begleiten. Die Reorganisation bzw. Neuausrichtung bezieht sich auf die Ebenen Strategie, Prozess sowie Informations- und Kommunikationstechnologie. Dabei werden einerseits die Potenziale und Restriktionen der Informations- und Kom- 
munikationstechnologie systematisch analysiert und frühzeitig in die Strategiebildung einbezogen, da sie Rahmenbedingungen für die Strategiebildung setzen. Andererseits werden mit Hilfe entsprechender Methoden Geschäftsstrategien entwickelt, deren Umsetzung bis auf die Ebene der Informations- und Kommunikationssysteme verfolgt wird, damit sie wirksam werden [Österle 1995, S. 23-24]. Das Vorgehensmodell des Business Engineering hat sich in verschiedenen Projekten bewährt [vgl. Bach et al. 1999, Österle et al. 2000]. Damit begründet sich nicht nur die Relevanz der Gestaltungsebenen, sondern auch deren Wiederverwendung zur Unterstützung der Neuausrichtung der Banken auf das Informationszeitalter.

Die strategische Ebene der Bankenarchitektur wird mit Hilfe eines Geschäftsmodells präzisiert, das sich durch definierte Merkmale und deren Ausprägungen beschreiben lässt. In Abbildung 2 werden Geschäftsmodelle durch Würfel dargestellt (vgl. Abschnitt 4.1). Die Kanten des Würfels veranschaulichen die Mehrdimensionalität der bestimmenden Merkmale eines Geschäftsmodells. Dies sind beispielsweise Kundenzielgruppen, Markenstrategie, Kernkompetenzen oder Ziele/Erfolgsfaktoren. Innerhalb eines solchen Würfels kann jedes Geschäftsmodell durch einen eigenen Ausschnitt dargestellt werden, der die entsprechenden Marken, Kundenzielgruppen, Vertriebswege usw. verkörpert. Der vollständig gefüllte Würfel veranschaulicht die traditionelle Universalbank, die anstrebt, weitgehend alles selbst anzubieten. Demgegenüber stellen die beiden nur teilweise ausgefüllten Würfel eine Direktbank oder eine Transaktionsbank dar, die nur einen Ausschnitt der Wertschöpfungskette selbst erfüllen. Da eine Direktbank (oder Logistikbank) beispielsweise nur ausgewählte Produkte anbietet, wird auch nur ein Teil der Prozesse benötigt. Die Prozesse werden dabei durch bestimmte Applikationen unterstützt, wobei jede Applikationen ihrerseits durch bestimmte Softwarebausteine realisiert wird [Leist/Winter 1999b, S. 5-6].

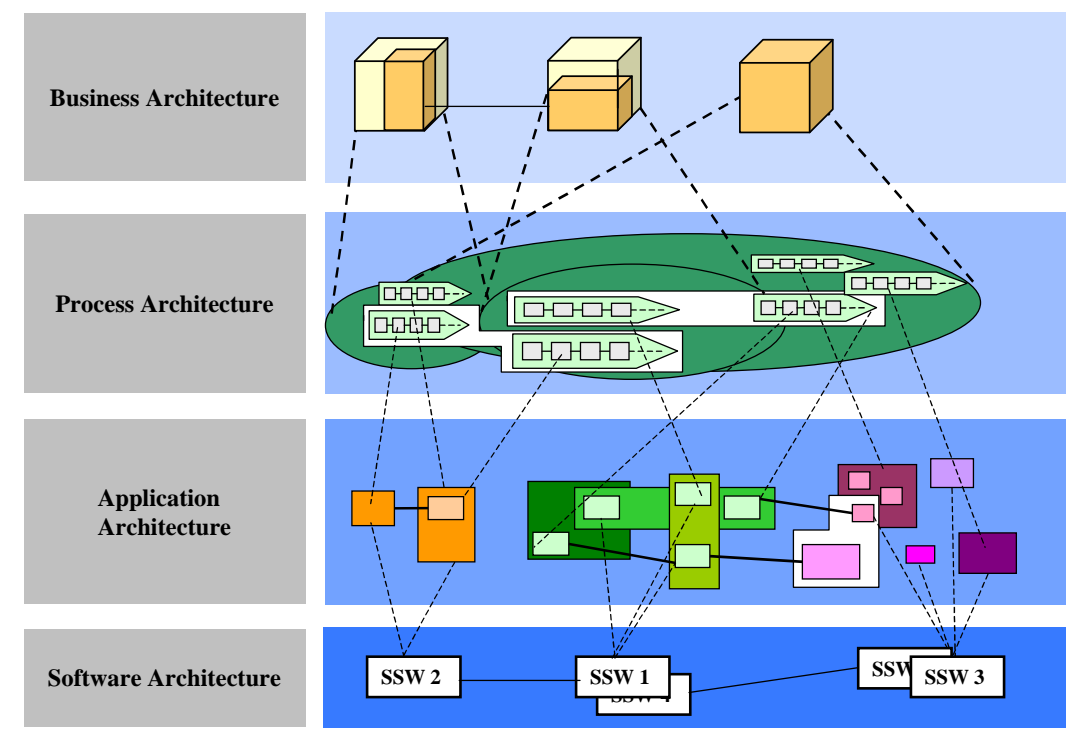

Abbildung 2: Bankenarchitektur [in Anlehnung an Leist/Winter 1999b, S. 5] 


\subsection{Bankenarchitekturen als Grundlage für das Geschäftsnetzwerk}

In ähnlicher Weise wie das vorgestellte Konzept der Bankenarchitektur eine Unterscheidung der Universalbank und der Transaktionsbank vornehmen kann, wird die Bankenarchitektur für einen Service Integrator grundlegend von einer Architektur für den Shared Service Provider bzw. für den Exclusive Service Provider differieren. Dies zeigen auch deutliche Unterschiede in den Schwerpunkten der Geschäftsmodelle: Während beispielsweise der Service Integrator eher Produkte im Bündel und an Ereignissen bzw. Lebenssituationen des Kunden orientiert anbietet, werden Exclusive und Shared Service Provider eher (Einzel-)Produkte anbieten (siehe hierzu auch Abschnitt 4.1).

Darüber hinaus unterscheiden sich die Rollen natürlich auch hinsichtlich der Prozesse und der Applikationen. So wird beispielsweise ein Schwerpunkt der Prozesse des Service Integrators in der Beratung und Betreuung seiner Kunden liegen, während sich viele Shared Service Provider eher auf Abwicklungs- und Abrechnungsprozesse beschränken. Entsprechend steht für den Service Integrator die Architektur seiner Kanalapplikationen im Vordergrund, während sich der Shared Service Provider eher auf die Architektur der Produktbestandssysteme ausrichtet. Die Erläuterungen verdeutlichen, dass für jede Rolle auf jeder Ebene der Bankenarchitektur ein eigenes Referenzmodell zu entwickeln ist. Auf diese Weise unterliegt jeder Rolle eine eigene Bankenarchitektur (siehe Abbildung 3).

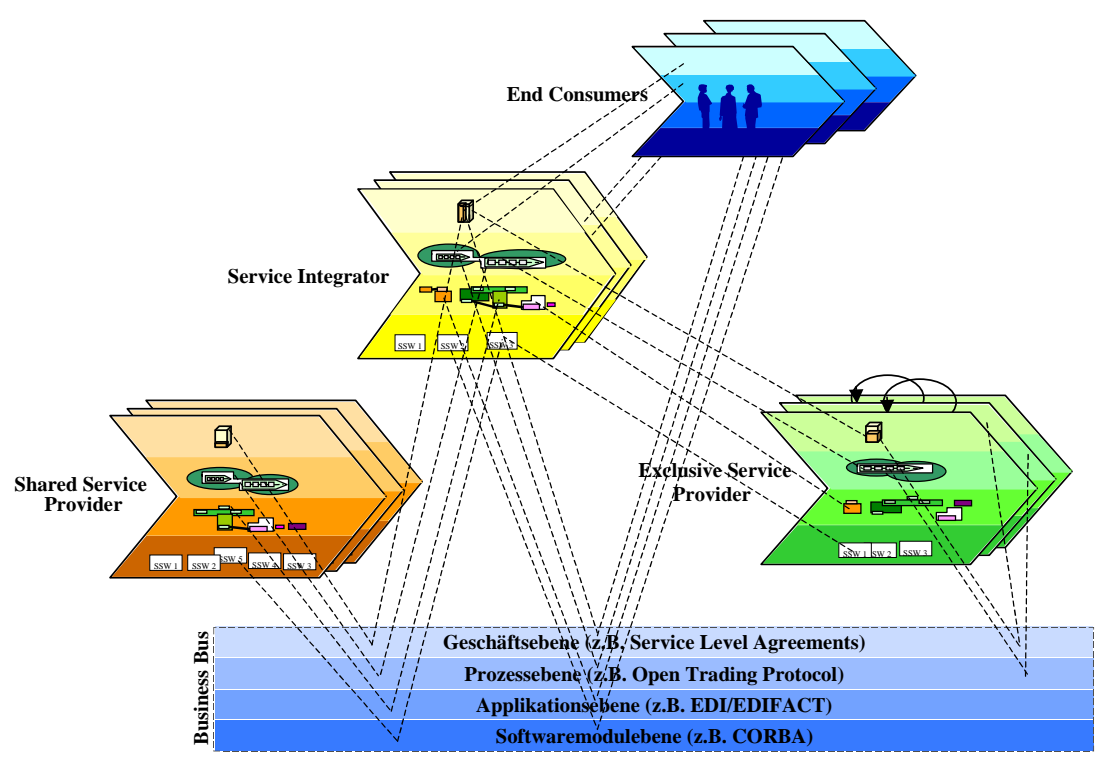

Abbildung 3: Bankenarchitektur als Grundlage für das Geschäftsnetzwerk [Leist/Winter 2000b] 
Gleichzeitig zeigt Abbildung 3, dass sich die Ebenen der Bankenarchitektur auch im Business Bus wiederfinden. Der Business Bus ist Grundlage für die Kommunikation und den Austausch von Leistungen zwischen den Providern, den Integratoren und den End Consumern. Dabei ist eine Kommunikation bzw. ein Leistungsaustausch über den Business Bus nur dann möglich, wenn die Beteiligten über gleiche Standards bzw. Protokolle verfügen. In der Schicht der Softwaremodule bzw. der Applikationen existieren schon jetzt eine Vielzahl verschiedener Standards (Corba, TCP/IP, EDI/EDIFACT, S.W.I.F.T. usw.), die den Datenaustausch ermöglichen.

Auf der Prozessebene gewährleisten Protokolle wie beispielsweise die Open Trading Protocols (OTP) die Integration von Geschäftsprozessen. Die OTP fokussieren auf die Business-to-Consumer-Beziehung und spiegeln die Prozesse der ,realen Welt' wider. Dabei werden standardisierte Prozessbausteine definiert (wie beispielsweise Kauf, Erstattung, Einzahlung, usw.), mit deren Hilfe die Geschäftsprozesse der Business-to-Consumer-Beziehung modelliert werden. Mit Hilfe dieser Transaktionsbausteine (die z.B. noch weiter hinsichtlich der auszutauschenden Daten spezifiziert werden) können die Schnittstellen zwischen den Prozessen des Service Integrators und des Kunden exakt definiert werden.

Die Kooperation zwischen Unternehmen bzw. die Geschäftsbeziehung zwischen Unternehmen und Kunde setzt ein gemeinsames Verständnis über die zu erbringende Leistung, Lieferzeiten und dergleichen voraus. Die Zusammenarbeit ist somit nur möglich, wenn sich die Partner auch auf dieser Ebene über die Nutzung eines gemeinsamen Protokolls (im Sinne von Vereinbarungen) einig sind, das die Art der Leistung, Lieferzeiten etc. festlegt. Beispiele für die Bestandteile solcher Protokolle sind: Rahmenverträge über Nutzungsrechte, Haftungsfragen, Sorgfaltsund Geheimhaltungspflichten, Vereinbarungen zu Entgelten, Lieferverträge, Service-Level-Vereinbarungen, Definition des grundsätzlichen Vertragsgegenstandes (z.B. Software-Erstellung) oder Qualitätsstandards.

\subsection{Zielsetzung des Kompetenzzentrums}

Die Zielsetzungen des Kompetenzzentrums bestehen darin, Referenzmodelle für jede Architekturebene zu erarbeiten, die eine Zusammenarbeit unabhängiger spezialisierter Banken in einem Unternehmensnetzwerk ermöglichen, sowie Aussagen zu den Standards für den Business Bus zu erarbeiten. Mit Hilfe des Business Bus werden die Schnittstellen der Zusammenarbeit der Banken im Netz definiert. Dabei konzentrieren sich die Arbeiten im Kompetenzzentrum in einem ersten Schritt auf die Architektur des Service Integrators sowie die Schnittstelle zwischen Service Integrator und Kunde. 
Die Referenzmodelle stellen eine wichtige Grundlage dar, um die eingangs gestellten Fragen beantworten zu können. So erarbeiten die Geschäftsmodelle wesentliche Merkmale der Rollen des Bankennetzwerks und können damit in umgekehrter Weise auch die Zuordnung verschiedener Banken zu den einzelnen Rollen anhand ihrer Merkmalsausprägungen unterstützen (siehe auch Abschnitt 4.1). Inwiefern bestehende, unternehmensspezifische Prozess- und Applikationsmodelle grundsätzlich neu entworfen werden müssen, wird sich ebenfalls mit der Entwicklung der Referenzmodelle feststellen.

\subsection{Vorgehensweise und Untersuchungsmethodik des Kompetenzzentrums}

Im Kompetenzzentrum wird zunächst die der Untersuchung zugrunde liegende Bankenarchitektur in Form eines Metamodells beschrieben, d.h. alle Elementtypen sowie deren Beziehungstypen werden für jede Ebene (Geschäftsmodelle, Prozesse und Applikationen) definiert.

Für die Ebene der Geschäftsmodelle gibt es bislang nur informale Beschreibungssprachen, die wenig ausgereift sind. Hierzu werden im Forschungsprojekt in Zusammenarbeit mit den Partnerunternehmen eigene Ansätze entwickelt. Als Beschreibungssprache für die Ebene der Prozesse bieten sich die ereignisgesteuerten Prozessketten (EPK) [Keller/Teufel 1997, S. 158-175] oder die Aufgabenkettendiagramme [Österle 1995, S. 95-96] aus der Business-Engineering-Vorgehensmethode PROMET [Österle 1995, S. 31] an. Die Erhebung der Applikationsarchitektur kann methodisch mit dem Business Systems Planning [Zachmann 1982, S. 31-53] unterstützt werden, womit neben den Applikationen auch Datenflüsse zwischen den Applikationen identifiziert werden. Die endgültige Auswahl der Beschreibungssprachen für jede Ebene der Bankenarchitektur sowie die Auswahl der Modellierungsmethoden wird allerdings im Projekt in Absprache mit den beteiligten Partnerunternehmen getroffen. Ausschlaggebend für diese Entscheidungen werden insbesondere die Erfahrungen der Partnerunternehmen mit den entsprechenden Beschreibungssprachen bzw. Methoden in ihren eigenen Projekten sein [Leist/Winter 2000a, S. 162].

In Zusammenarbeit mit den am Projekt beteiligten Partnerunternehmen werden anschließend die betreffenden unternehmensspezifischen Modelle fokussiert auf den Bereich des Service Integrators erhoben [Leist/Winter 1999b, S. 6-7]. Die Einschränkung erfolgt zugunsten einer tiefergehenden Untersuchung. Auf diese Weise wird eine Validierung des Metamodells vorgenommen. Auf der Basis der unternehmensspezifischen Modelle können zunächst Aussagen zu einer Good Practice gemacht werden. Darüber hinaus werden mit Hilfe von Schwachstellenanalysen und verschiedenen Kreativitätstechniken für jede Ebene Referenzmodelle abgeleitet. In Abbildung 4 werden die drei verschiedenen Modell-Typen MetaModell, unternehmensspezifisches Modell und Referenzmodell grafisch abgebildet. 

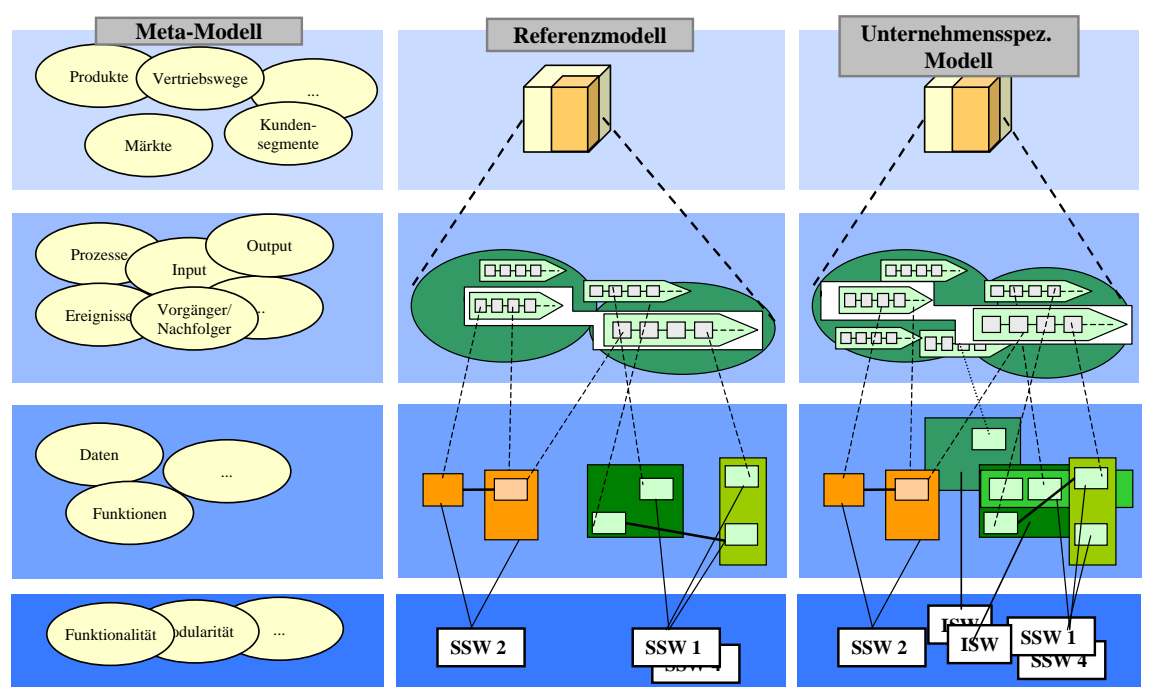

Abbildung 4: Untersuchungsmethodik [Leist/Winter 1999b, S. 7]

In einem zweiten Schritt wird darauf aufbauend, ebenfalls in Zusammenarbeit mit den Partnerunternehmen, ein Vorgehensmodell auf der Grundlage von Good Practices entwickelt, das Hinweise für die konsistente Gestaltung von Geschäftsmodellen, Prozessen und Applikationen gibt und eine Ganzheitlichkeit des Ansatzes gewährleistet [Leist/Winter 1999b, S. 6-7]. Der Schwerpunkt der aktuellen Projektarbeiten besteht in der Definition der Metastruktur sowie in der Erhebung erster unternehmensspezifischer Modelle.

\subsection{Fokussierung auf die Rolle des Service Integrators}

Im Zentrum der Aktivitäten des Service Integrators steht die Intensivierung der Beziehung zum Kunden. Durch fehlende Koordination und Integration der Vertriebswege einer Bank ist es allerdings oftmals nicht möglich, die Beziehung zum Kunden ganzheitlich zu pflegen und zu unterhalten, da keine „einheitliche Sicht“ auf den Kunden (jeder Vertriebsweg kennt nur seinen Teil der Kundenbeziehung) existiert. Die Vertriebswegesteuerung und deren Ziel eines optimalen Flusses von Produkten, Dienstleistungen und Informationen über die Distributionswege [in Anlehnung an Schlögel 1997, S. 14-21] muss somit als Grundvoraussetzung für die Etablierung als Service Integrator gesehen werden (optimal kann hier unter vielerlei Gesichtspunkten (z.B. Zeit, Kosten, Wertschöpfung, Qualität, Flexibilität) verstanden werden und ist im konkreten Fall mit den Merkmalen des Geschäftsmodells eines Unternehmens in Einklang zu bringen). Bei der Frage, welche Aufgaben im Rahmen der Vertriebswegesteuerung zu definieren sind, orientieren sich die Arbeiten im CC BAI ebenfalls auf die Gestaltungsebenen der Bankenarchitektur. Abbildung 5 stellt die Themenfelder dar. 


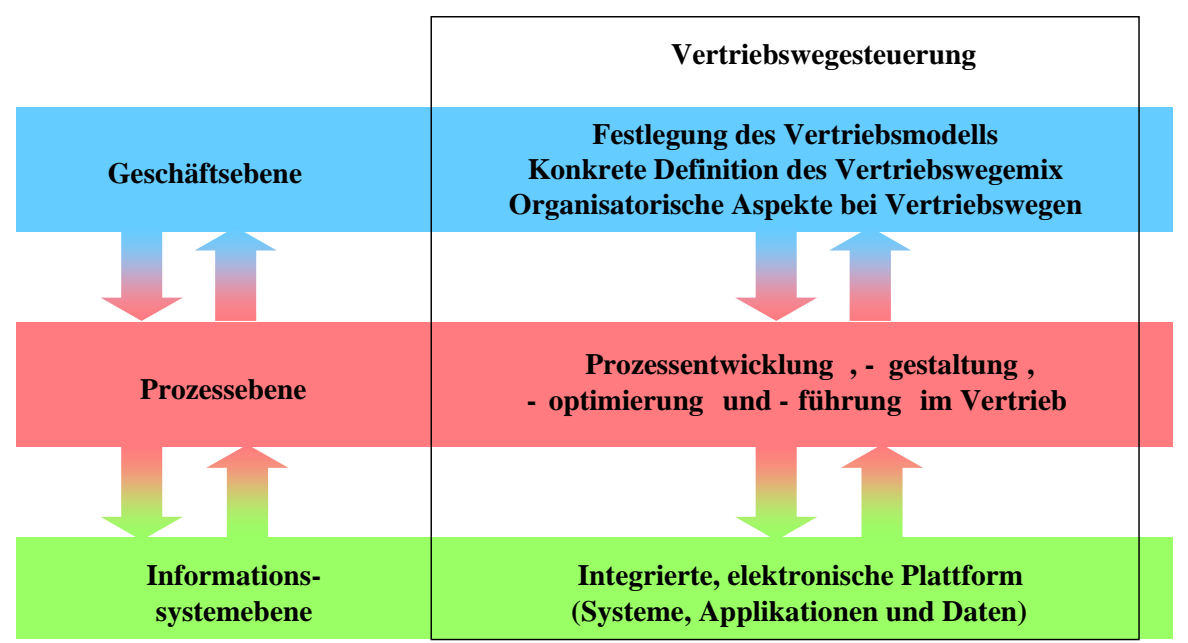

Abbildung 5: Aufgabenfelder im Rahmen der Vertriebswegesteuerung

Die Graphik zeigt, dass auf Geschäftsebene beispielsweise darüber entschieden werden muss, wie viel Konkurrenz sich die einzelnen Vertriebswege gegenseitig machen sollen. Extrempositionen sind dabei einerseits die Differenzierungsstrategie, bei welcher einzelne Vertriebswege völlig unabhängig von anderen Kanälen agieren können (marktliche Koordination) und andererseits die Integrationsstrategie, bei der durch eine zentrale Stelle die gesamte Steuerung des Marktauftritts erfolgt (hierarchische Koordination). Das Ergebnis der Arbeiten sollte hierbei sein, unter Vermeidung der Kannibalisierung der Vertriebswege trotzdem eine gewisse Konkurrenzsituation zu etablieren, um zusätzlich zur oben genannten Intention auch Formalzielsetzungen (z.B. Effizienz usw.) erreichen zu können. Nach dieser grundlegenden Entscheidung über die organisatorische Einordnung kann mit der Entwicklung des Marktauftritts (z.B. „die Beraterbank“) begonnen werden. Die Konkretisierung dieses Marktauftritts spiegelt sich dem gegenüber im Vertriebswegemix wider, der differenziert auf die fachliche Konzeption eingeht, d.h. welches Produkt zu welchem Preis, mit welchen Serviceleistungen usw. über welchen Vertriebsweg angeboten wird. Marktauftritt und Vertriebswegemix fließen dabei in aggregierter Form als wesentliche Merkmale in das Geschäftsmodell des Service Integrators ein (vgl. Abschnitt 4).

In den unteren beiden Ebenen sind auf Basis der Definition zunächst Prozesse neu bzw. weiter zu entwickeln, um auf die geänderten Anforderungen einzugehen. Andererseits ist auf Ebene der Informationssysteme eine elektronische Plattform zu verwirklichen, die sowohl Systeme, Applikationen und Daten logisch und technisch integriert. Gerade beim letzten Aspekt spielen Schnittstellenstandards oder Abstraktionsschichten (z.B. Middleware), wie sie im Zusammenhang mit dem Business Bus angedacht werden, eine wesentliche Rolle. 


\section{Das Geschäftsmodell als strategische Ebene der Bankenarchitektur}

Im diesem Abschnitt werden erste Ergebnisse des Kompetenzzentrums vorgestellt. Dabei werden in Abschnitt 4.1 die Merkmale und Merkmalsausprägungen eines Geschäftsmodells vorgestellt und anschließend an einem Beispiel erläutert. Dies wird auch zeigen, inwiefern die Merkmalsausprägungen Schlüsse über die Zuordnung zu einer Rolle zulassen. Der anschließende Abschnitt 4.2 konkretisiert das Geschäftsmodell für die Rolle des Service Integrators.

\subsection{Positionierung in Unternehmensnetzwerken auf der Grundlage von Geschäftsmodellen}

\subsubsection{Merkmale und Ausprägungen des Geschäftsmodells}

Das Geschäftsmodell wird in der Literaturmeinung nicht als feststehender Begriff verstanden, sondern verkörpert vielmehr eine Vorstellung davon, wie sich das Unternehmen gegenüber den sozioökonomischen Systemen (Kunden, Lieferanten, Konkurrenten usw.) verhält, mit denen es in Berührung kommt [vgl. hierzu Österle 1996, S. 447-449; Timmers 1998, S. 3-8; Viscio/Pasternack 1996; Deutsche Bank AG 1999; Fugmann et al. 1999, S. 255-258].

Die Einordnung erfolgt dabei meistens in den Gesamtkontext des Geschäftsoder Businessplans, der neben dem Betrachtungsgegenstand Geschäftsmodell auch die rechtlichen Verhältnisse, den Kapitalbedarf und die -verwendung, sowie Chancen und Risiken des Unternehmens enthält. Im Gegensatz zu diesen generellen Fragen des Unternehmensgegenstandes (z.B. Rechtsperson), beschreibt das Geschäftsmodell in erster Linie die Außensicht auf die Unternehmenssituation, d.h. die Positionierung des Unternehmens in den fokussierten Märkten. Daneben wird jedoch auch die Innensicht in Form der primären Wertschöpfungsaktivitäten betrachtet, d.h. die Gewinnung, Produktion, Bereitstellung und der Vertrieb von Gütern und Dienstleistungen. Hierbei spielen die unternehmenseigenen Potenziale und Kompetenzen eine wesentliche Rolle.

Im Folgenden wird ein erster Ansatz für die Beschreibung eines Geschäftsmodells für den Bankenbereich vorgestellt. Der Schwerpunkt liegt dabei in der Herleitung der Merkmale, mit denen charakteristische Eigenschaften der Banken festgelegt werden:

1. Außensicht

Die Außensicht umschließt, auf welchem Markt das Unternehmen tätig sein will, d.h. mit welchen Produkttypen/Prozessklassen das Unternehmen den Bankenmarkt „,bedienen“ will und welche Endkunden in welchen Regionen letzt- 
endlich angesprochen werden sollen. Dabei sind die Produkttypen/ Prozessklassen nicht alleine nach ihrer Art (Kredit, Anlage usw.), sondern auch dahingehen zu unterscheiden, ob sie einzeln, in einem Bündel oder ausgerichtet an den Kundenprozessen angeboten werden. Abhängig davon sind auch die Festlegung der Marke sowie die Auswahl des Vertriebswegs und des Vertriebskonzepts. Mit Außensicht wird somit im Wesentlichen der Marktauftritt einer Bank bestimmt. (Merkmale sind beispielsweise: Land/Region, Kunde, Kernprodukte und Leistungen, Eigenschaft der Wertekette, Marke, Vertrieb.)

2. Innensicht

Die Innensicht beschreibt die Kompetenzen und Stärken des Unternehmens, die zur Umsetzung der Außensicht notwendig sind. Orientierung und Maßgröße bieten die Ziele und Erfolgsfaktoren des Unternehmens. Darüber hinaus sind Kooperationen und die Organisationsstruktur (eher zentrale oder eher dezentrale Ausrichtung) des Unternehmens zu berücksichtigen. (Merkmale sind beispielsweise: Kompetenzfelder, Ziele-/Erfolgsfaktorensegmente, Kooperationen, Organisationsstruktur.)

Mit diesen Merkmalen und der Unterscheidung in eine Außen- und Innensicht wird eine Strukturierung der in den meisten Unternehmen nur in Fließtext vorhandenen Geschäftsmodelle vorgenommen. Dabei werden nicht nur Aussagen zur strategischen Ausrichtung des Unternehmens festgelegt, sondern auch Aussagen, die Rückschlüsse auf die Position des Unternehmens im Netzwerk erlauben. Die Ausführungen zu den Merkmalen des Geschäftsmodells können in dieser Form allerdings noch keinen Anspruch auf Vollständigkeit erheben. Ein Nachweis über die Vollständigkeit erscheint nur empirisch möglich. Dennoch werden auf der Grundlage der folgenden Beispiele erste Hypothesen im Hinblick auf die Aussagekraft ausgewählter Merkmale zur Rolle der Unternehmen im Geschäftsnetzwerk des Informationszeitalters formuliert.

\subsubsection{Anwendung des Geschäftsmodells zur Charakterisierung von Unternehmen}

Ein Discount Broker (z.B. youtrade der Credit Suisse www.youtrade.ch oder ConSors www.consors.de) stellt dem Endkonsumten Dienstleistungen rund um den Wertpapierhandel zur Verfügung. Sie werden Retailkunden oder vermögenden Privatkunden angeboten, die ein Depot bei dem Broker eröffnet haben. Kunden können dabei sowohl Inländer wie Ausländer sein. Da der Handel ausschließlich über elektronische Vertriebswege (v.a. Telefon und Internet) durchgeführt wird und mit keiner individuellen und persönlichen Beratungsleistung seitens der Bank verbunden ist, wird er dem Kunden zu besonders günstigen Konditionen angeboten. Dem Kunden werden dabei Informationen zur eigenständigen Entscheidungsfindung offeriert.

Das Kernprodukt des Discount Brokers ist der Zugang zu den an der Börse gehandelten Wertschriften und Fonds nebst einem Reporting über durchgeführte Wertschriften-Transaktionen. Hinzu kommen (insbesondere bei dem Discount Broker Consors) sehr detaillierte Informationen u.a. über den Online-Handel, über 
rechtliche Vorschriften, zu möglichen Anlageprodukten und deren Bewertung sowie Erläuterungen zur Bildung von Anlageportefeuilles. Der Discount Broker bietet somit seinen Kunden in einem Paket vielfältige Informationen an, die ihn dazu befähigen, seine Börsengeschäfte selbstständig durchzuführen.

Die Erfolgsfaktoren für das Unternehmen bestehen in einer schnellen Übermittlung von Informationen, dem Angebot besonders günstiger Konditionen und einem hohen Sicherheitsstandard.

In Abbildung 6 ist das Geschäftsmodell eines Discount Brokers in vereinfachter Form graphisch dargestellt. Das Geschäftsmodell enthält sieben Merkmale (Land/ Region, Kunde, Marke usw.) und deren Ausprägungen (z.B. Inland, Euro-Inland, Euro-Ausland). Dabei zeigen die grau gefärbten Flächen an, welche Merkmalsausprägungen den Discount Broker beschreiben.

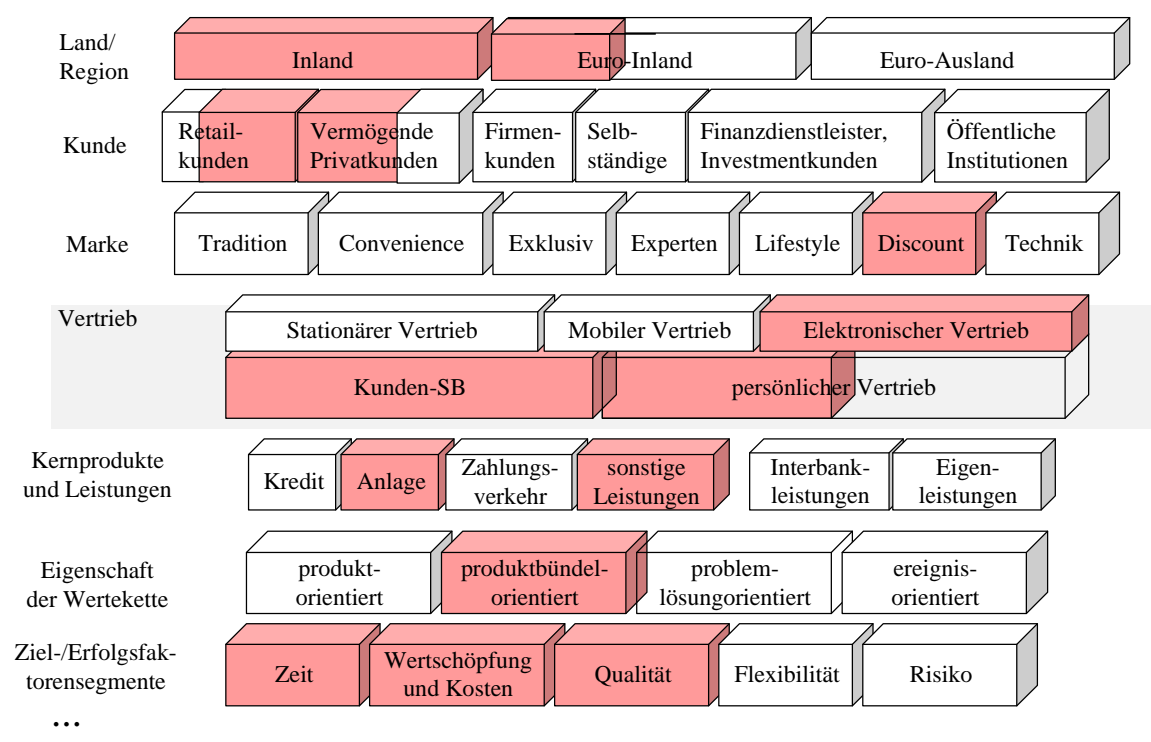

\section{Abbildung 6: Das Geschäftsmodell eines Discount Brokers}

Eine Kapitalanlagegesellschaft ist berechtigt, im eigenen Namen für gemeinschaftliche Rechnung der Einleger (Anteilinhaber) nach dem Grundsatz der Risikomischung Wertpapiere oder Grundstücke zu erwerben. Das bei einer Kapitalanlagegesellschaft gegen Ausgabe von Anteilscheinen eingelegte Geld und die damit angeschafften Wertpapiere und Bezugsrechte bzw. Grundstïcke und Erbbaurechte bilden ein Sondervermögen [Büschgen 1991, S. 385-386]. Anteilinhaber sind in der Regel Retail- oder vermögende Privatkunden sowie Finanzdienstleister und Investmentkunden im In- und Ausland. Die Kapitalanlagegesellschaft vermittelt dem Kunden, Experte bei der Auswahl beispielsweise der Aktien des Fonds zu sein. Dem Kunden bieten sich verschiedene Möglichkeiten, Anteile an dem Fonds zu erwerben. So kann der Kunde über Absatzmittler (wie beispielsweise einen Discount Broker (z.B. Consors) oder eine Bank) oder teilweise auch über die Kapitalanlagegesellschaft selbst Anteile beziehen. Dies ist in der Regel auf elektronischem Wege möglich. 
Die Kapitalanlagegesellschaft bietet als wichtigstes Produkt dem Kunden Anteile seines Fonds an. Kernprodukte sind somit Anlageprodukte. Ziel- und Erfolgsfaktorensegmente für die Kapitalanlagegesellschaft sind die Qualität und das Risiko-/Renditeverhältnis des Fonds.

In Abbildung 7 ist das Geschäftsmodell der Kapitalanlagegesellschaft graphisch dargestellt. Die grau getönten Flächen zeigen wieder an, welche Merkmalsausprägungen die Kapitalanlagegesellschaft beschreiben.

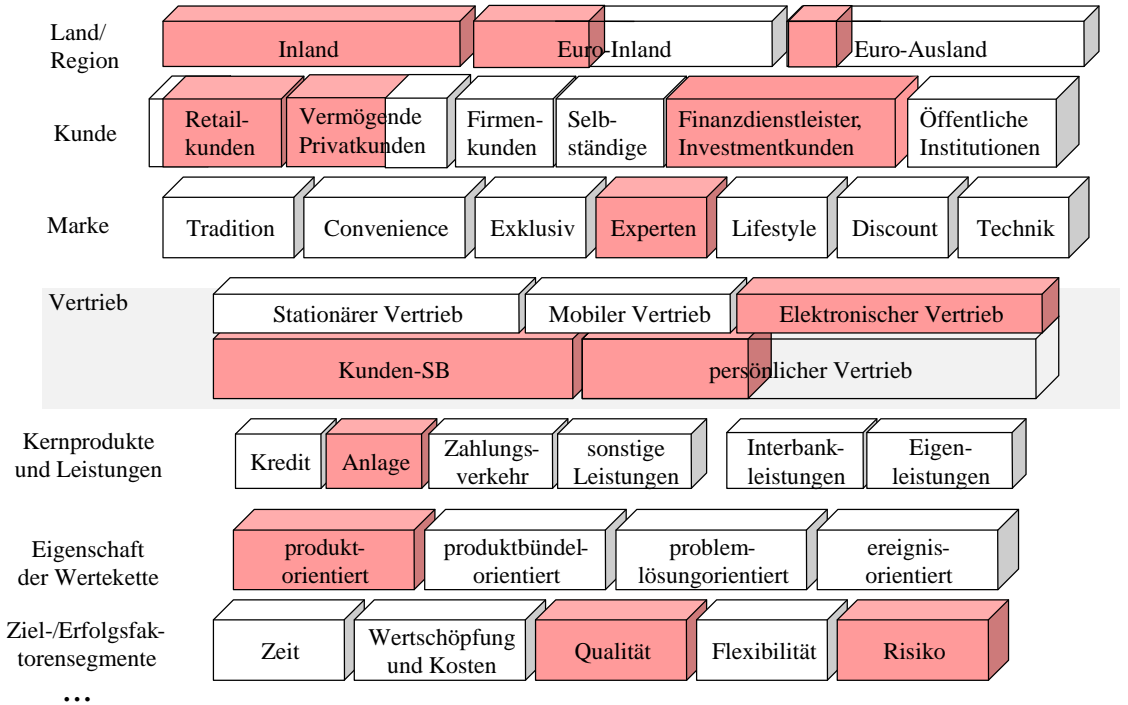

Abbildung 7: Das Geschäftsmodell einer Kapitalanlagegesellschaft

Beide Unternehmen sprechen Retail- und vermögende Privatkunden auf dem Inlandsmarkt an. Auf den ersten Blick deuten diese Merkmalsausprägungen darauf hin, dass beide Unternehmen die Rolle des Service Integrators einnehmen. Allerdings zeigt die Einordnung der Unternehmen hinsichtlich des Merkmals Vertrieb, dass beide Unternehmen ihren Schwerpunkt auf die Kundenselbstbedienung mittels elektronischem Vertriebsweg legen und somit nicht auf persönliche Betreuung bzw. Bedienung des Kunden setzen. Während der Discount Broker diesen Schwerpunkt explizit wählt, da er auf eine Kundengruppe abzielt, die selbstständig und kostengünstig Wertpapiere handeln möchte (Markenstrategie: Discount), lässt sich dies für die Kapitalanlagegesellschaft nicht so eindeutig bestimmen. Die Kapitalanlagegesellschaft versucht dagegen eher ein breites Spektrum der Privatkunden abzudecken. Das Angebot verschiedener Vertriebswege oder ein größeres Gewicht auf die persönliche Ansprache durch einen Kundenberater ist für die Kapitalgesellschaft dennoch nicht notwendig, da neben ihrem eigenen Vertrieb weitere Vertriebswege über Banken und Finanzdienstleister bestehen, die dann auch die persönliche Ansprache des Kunden übernehmen. Dies deutet darauf hin, dass die Kapitalanlagegesellschaft die Rolle eines Service Integrators nur in Teilen wahrnimmt. Ein weiteres Indiz dafür ist, dass sie nur einzelne Produkte und nicht an den Bedürfnissen des Kunden ausgerichtete Produktbündel oder Problemlösun- 
gen anbietet. Insofern nimmt die Kapitalgesellschaft eher die Rolle eines Shared Service Provider wahr, der seine Produkte (Anteile an seinen Fonds) verschiedenen Service Integratoren anbietet.

\subsection{Fokussierung des Geschäftsmodells für die Rolle des Service Integrators}

Die wesentliche Aufgabe des Service Integrators besteht darin, eine Beziehung zum Kunden zu pflegen, um ausgehend von seinen Bedürfnissen und Problemen eine adäquate Lösung anzubieten. Aus Sicht des Kunden wird der Service Integrator somit insbesondere durch die Merkmale des Geschäftsmodells beschrieben, die seinen Marktauftritt charakterisieren. Die Frage, wie diese Merkmale des Service Integrators letztlich ausgestaltet werden können, soll im Folgenden detaillierter untersucht werden.

In der Literatur sind zum Entwurf einer Vertriebs- oder Marketingkonzeption eine Reihe von Vorgehen und Methoden vorgestellt und diskutiert worden [vgl. für allgemeingültige Vorgehen Kotler/Bliemel 1995; Becker 1993; Belz 1998, auf den bankbetrieblichen Sektor bezogene Ausführungen publizierten zu Teilaspekten Schneider 1997; Schierenbeck 1999; Stäger 1999]. Ähnlich wie Schlögel fordern dabei auch viele andere Autoren eine Orientierung des Distributionssystems an den Kundenbedürfnissen als wesentliches Zielkriterium [Schlögel 1995, S. 18: Die gleichermaßen wichtige Bedingung einer Definition und Berücksichtigung der Wettbewerbs- und Konkurrenzposition des Unternehmens erfolgt in unserem Fall bereits in der vorgelagerten strategischen Phase]. Wie ist jedoch eine solche Ausrichtung der Distribution oder umfassender betrachtet der gesamten angebotenen Problemlösung an den Kundenbedürfnissen vorzunehmen?

Eine Möglichkeit, diese Fragestellung zu behandeln, ist die Analyse der Bedürfnisse und ihre logische Aufgliederung in Bestandteile. Diese sind zwar nicht unabhängig voneinander zu sehen, erlauben jedoch trotzdem ein präziseres Eingehen auf das Kundenproblem.

Eine Komponente der Bedürfnisse zeigt sich dabei im weitläufig diskutierten Wandel des Kundenverhaltens [vgl. Schierenbeck 1999, S. 5-9; Kurzmeyer 1999, S. 127-129; Eilenberger/Burr 1997, S. 192]. Der Kunde besitzt demzufolge in immer größerem Masse den Bedarf nach zeitlicher und räumlicher Unabhängigkeit, ist einerseits preis- und convenienceorientiert, bei anderen Leistungen wiederum äußerst anspruchsvoll und wählerisch und achtet auf qualitativ hochwertige Beratung. Eine Folge dieser Änderung und Vielfalt der Ansprüche sind verhaltens- und werteorientierte Segmentierungsansätze, wie sie bspw. von Gunnarsson [Gunnarsson 1999, S. 39-41, 113-167] aufgezeigt werden. Problematik vieler dieser Ansätze ist jedoch die mangelnde Operationalisierbarkeit, d.h. die Ableitung konkreter Maßnahmen, um auf einen „emanzipierten, informierten und kritischen“ Kunden einzugehen bzw. zu reagieren. In der Realität wird demzufolge das „Ausleben" solcher Kundencharakteristika meist nur durch ein möglichst breites und unspezifisches Leistungs- und Sortimentsangebot seitens der Bank unterstützt. 
Um dem entgegen zu wirken und damit die Individualisierung des Angebots voran zu treiben, ist als weiteres wesentliches Bedürfniselement der Kundenprozess [vgl. dazu auch Österle/Fleisch/Alt 2000, S. 45-50] (z.B. Erwerb von Wohneigentum), innerhalb dessen Bank- oder allgemein Finanzdienstleistungen benötigt werden, zu analysieren. Dieser zeigt auf, in welchem Umfeld und Zusammenhang der Kunde Leistungen zur Lösung seines konkreten Problems von der Bank oder von Drittanbietern erwartet und wie diese in ihrer Art (z.B. mit umfassender Betreuung) gestaltet werden müssen. Damit ist nicht nur das zuvor analysierte Verhalten bzw. die Wertvorstellung des Kunden besser zu berücksichtigen, sondern es kann bei der späteren Entwicklung der Problemlösung auch unter Einbindung neuer Kooperationspartner dem Kunden ein komplettes Leistungsangebot nach der Idee des Service Integrators bereitgestellt werden. Als dritter Kernpunkt ist die Untersuchung durchzuführen, welche Präferenzen der Kunde bezüglich der Form der konkreten Leistungsvermittlung und -inanspruchnahme besitzt, d.h. welche Hilfsmittel und Applikationen er zum Zugriff auf die Dienstleistung bevorzugt und wünscht [vgl. hierzu auch Schierenbeck 1999, S. 11-41]. Die nachfolgende Abbildung stellt die angesprochenen Ebenen dar.

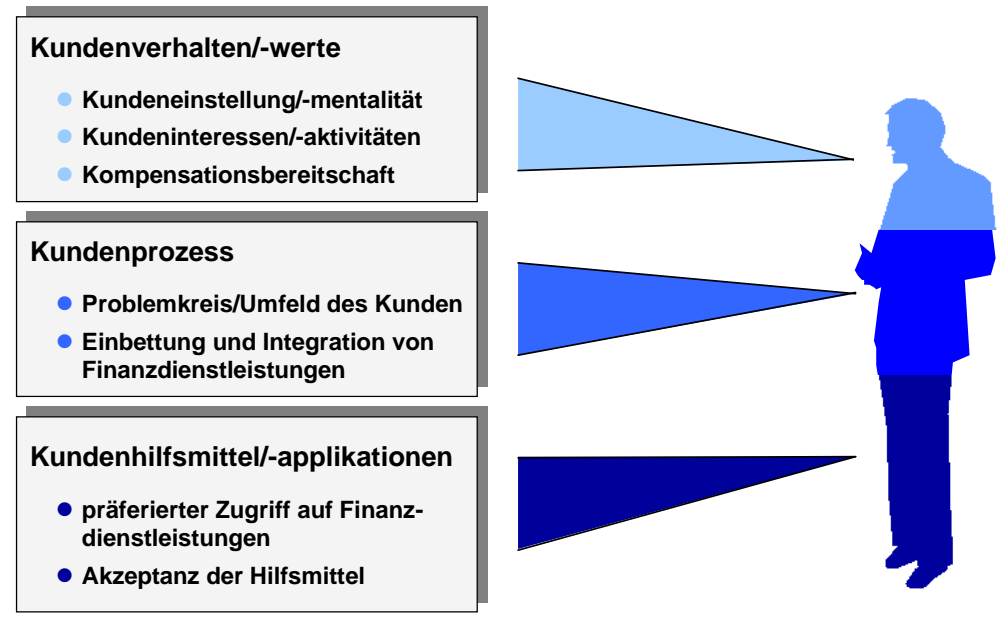

Abbildung 8: Mehrstufige Analyse des Kundenbedürfnisses

Abbildung 8 zeigt, dass die zu entwickelnde Problemlösung den Kunden sowohl „vom Kopf her“ ansprechen, d.h. mit seinem Verhaltensmuster und seiner Wertvorstellung korrespondieren, als auch seine Prozesse und Abläufe unterstützen bzw. vorantreiben muss. Um ihre Akzeptanz und den Erfolg auch im Sinne der Kundenbindung sicher zu stellen, muss sie letztlich auch auf dem von ihm präferierten Weg genutzt werden können.

Die vorgestellte Aufgliederung der Bedürfnisse stellt deshalb eine Basis für das Gestalten der Problemlösung dar, d.h. jede der Ebenen muss eine geeignete Entsprechung mittels Instrumente des Vertriebs- und Marketingmix finden (ebenso wichtig sind daneben natürlich originäre Zielsetzungen, wie die Rentabilität oder 
das Geschäftsrisiko des Unternehmens). Dabei ist nicht nur die Wirkung der Instrumente auf die entsprechende Ebene der Kundenbedürfnisse zu untersuchen, sondern auch deren gegenseitige Abhängigkeit zur Entwicklung eines konsistenten „Bildes“ der Lösung.

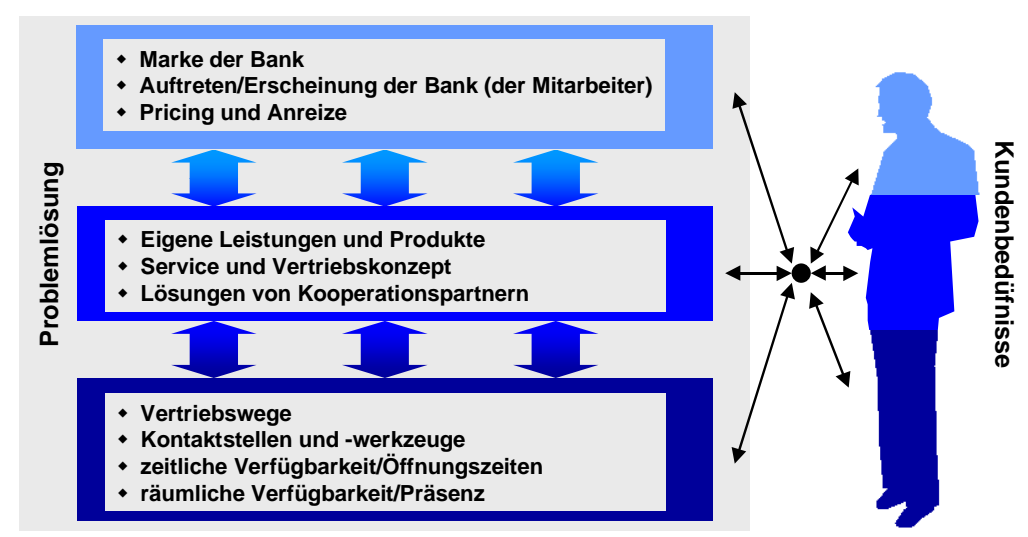

Abbildung 9: Mehrstufiger Aufbau der Problemlösung

Die Abbildung 9 veranschaulicht die Zusammenhänge. Dabei werden an oberster Stelle Instrumente genannt, die vornehmlich die Werte und Einstellungen des Kunden ansprechen sollen, um dem eigentlichen Leistungsbezug eine ,positive Grundstimmung“" zu unterlegen (,Senkung und Abbau von Widerständen hinsichtlich der Kaufbereitschaft“" [vgl. Büschgen 1979, S. 34]). Sind diese Rahmenbedingungen gesetzt, werden bezogen auf den Kundenprozess eigene und fremde Leistungen und Produkte dem Kunden mit einem adäquaten Service und Vertriebskonzept angeboten. Um letztlich den Zugriff des Kunden auf die Leistungen zu unterstützen, müssen sich der Einsatz und die Charakteristik der Vertriebswege und Kontaktstellen an den Kundenpräferenzen sowie an den Eigenschaften der jeweils angebotenen Leistungen (im Kundenprozess) orientieren.

Aufbauend auf der oben vorgestellten Analyse der Kundenbedürfnisse kann nunmehr in Zusammenarbeit mit den Partnerunternehmen eine Problemlösung entwickelt werden, die insbesondere den letzten Aspekt einer optimalen Distribution von Leistungen im Kundenprozess als Schwerpunkt besitzt. 


\section{Zusammenfassung}

Im vorliegenden Beitrag wurden zunächst die Potenziale (Reduktion der Komplexität und Fokussierung auf Kernkompetenzen, Kostenvorteile durch Bildung groBer Transaktionsbanken, erhöhte Flexibilität bei der Generierung von Problemlösungen durch Bildung kleiner Vertriebsbanken) einer Kooperation spezialisierter Banken diskutiert. Darauf aufbauend wurde die Vision eines Geschäftsnetzwerkes der Banken vorgestellt. Dabei wurden die verschiedenen Rollen der Bankdienstleister in Netzwerken identifiziert (Service Integrator, Shared Service Provider, Exclusive und Service Provider) und bankbetrieblichen Funktionen zugeordnet. Mit der Beschreibung der Vision bleibt offen, welche technischen und organisatorischen Maßnahmen zu treffen sind, damit Banken in einem Netzwerk zusammenarbeiten können. Diese Frage wird im Kompetenzzentrum Bankenarchitekturen im Informationszeitalter aufgegriffen. Die Ziele des Kompetenzzentrums bestehen darin, Referenzmodelle für spezialisierte Banken zu entwickeln und ein Vorgehensmodell zur Anpassung der Ist-Situation auf Grundlage der Referenzmodelle zu erarbeiten. Das Kompetenzzentrum arbeitet mit sechs Partnerunternehmen in einem Zeitraum von zwei Jahren zusammen. Erste Ergebnisse des Kompetenzzentrums wurden in diesem Beitrag vorgestellt. Zum einen wurde aufgezeigt, wie die Merkmale und Merkmalsausprägungen des Geschäftsmodells verwendet werden, um diejenige Rolle die Unternehmen im Visionsmodell einnehmen bzw. einnehmen könnten zu identifizieren. Zum anderen wurde eine Detaillierung des Geschäftsmodells für die Rolle des Service Integrators vorgenommen und dabei als wesentliche Merkmale des Geschäftsmodells die Konkretisierung des Marktauftritts sowie die Entwicklung des Vertriebswegemix beispielhaft beschrieben. 


\section{Literatur}

[Bach/Vogler/Österle 1999]

Bach, V., Vogler, P., Österle, H., Business Knowledge Management: Praxiserfahrungen mit Intranet-basierten Lösungen, Springer, Berlin et al., 1999

[Becker 1993]

Becker, J., Marketing-Konzeptionen: Grundlagen des strategischen Marketing-Managements, Vahlen, München, 1993

[Belz 1998]

Belz, C., Akzente im innovativen Marketing, Thexis, St.Gallen, 1998.

[Brenner 1995]

Brenner, C., Techniken und Metamodell des Business Engineering, Dissertation an der Universität St.Gallen, 1995

[Büschgen 1979]

Büschgen, H. E., Bankbetriebswirtschaftslehre, Gabler, Stuttgart/New York, 1979

[Büschgen 1991]

Büschgen, H.E., Das kleine Börsen-Lexikon, Wirtschaft und Finanzen, Düsseldorf, 1991

[Choi 1997]

Choi, S., Strategien von Banken im globalen Wettbewerb, Wiesbaden, DT. Universitätsverlag, 1997

[Deutsche BankAG 1999]

o.V., „Wie erstelle ich einen Business-Plan?“, Anleitung der Deutschen Bank AG, Wirtschaftswoche, (http://www.wiwo.de/economy/businessplan.htm)

[Eilenberger/Burr 1997]

Eilenberger, G., Burr, W., Zur Virtualisierung von Banken: Konsequenzen des Electronic Banking für Bankorganisation und Bankwettbewerb, in: Hörter, S., Wagner, A.: Visionen im Bankmanagement: Zukünftige Anforderungen an die Führung von Banken, Beck, München, 1997, S. 183-215

[Fleisch/Klüber/Österle 1999]

Fleisch, E., Klüber, R., Österle, H., Development of a Method for Inter-Business Networking, in: Pries-Heje, J. et al. (Eds.): Proc. 7th European Conference on Information Systems, Volume 3, Copenhagen Business School, 1999, 775-783

[Fugmann/Heinrich/Leist/Winter 1999]

Fugmann, T., Heinrich, B., Leist, S., Winter, R., Banking im Informationszeitalter Formen und Gestaltungsfragen von Wertschöpfungsnetzwerken im Bankbereich, in: Steiner, Manfred et al. (Hrsg): Elektronische Dienstleistungswirtschaft und Financial Engineering, Schüling Verlag, Münster, 1999, S. 237-261

[Gunnarsson 1999]

Gunnarsson, J., Portfolio-Based Segmentation and Consumer Behavior, Stockholm, Elanders Gotab, 1999

[Hagel/Singer 1999]

Hagel, J., Singer, M., Unbundling the Corporation, in: Harvard Business Review, March-April 1999, S. 133-141

[Keller/Teufel 1997]

Keller, G., Teufel, T., SAP R/3 prozessorientiert anwenden: Iteratives Prozess-Prototyping zur Bildung von Wertschöpfungsketten, Bonn et al. 1997 
[Kotler/Bliemel 1995]

Kotler, P., Bliemel, F., Marketing-Management: Analyse, Planung, Umsetzung und Steuerung, Schäffer-Poeschel, Stuttgart, 1995

[Kurzmeyer 1999]

Kurzmeyer, H., Internet Banking - Konzepte und Marktstrategien der Credit Suisse, in: Basler Bankvereinigung (Hrsg.): Multi-Channel Distribution im Banking, Tagungsband zum 6. Basler Bankentag, Paul Haupt, Bern, 1999, S. 126-139

[Leist/Winter 1999a]

Leist, S., Winter, R., Component-Based Banking - Modularization of Information Processing in Banks as a Foundation for Virtual Business, in: Abramowicz, W. (ed.): BIS'99, 3rd International Conference on Business Information Systems, Poznan, Poland, 14-16 April 1999,Practical Sessions, pp. 197-208

[Leist/Winter 1999b]

Leist, S., Winter, R., Banking of the Information Age - Vision, Transformation, and Design Principles, Arbeitsbericht (BE HSG/FP BAI/01) des Instituts für Wirtschaftsinformatik der Universität St.Gallen, April 1999

[Leist/Winter 2000a]

Leist, S., Winter, R., Finanzdienstleistungen im Informationszeitalter - Vision, Referenzmodell und Transformation, in: Belz, Christian; Bieger, Thomas (Hrsg.): Dienstleistungskompetenz und innovative Geschäftsmodelle, Thexis , St.Gallen, 2000, S. 150-166

[Leist/Winter 2000b]

Leist, S., Winter, R., Akquisitionsunterlagen zum Kompetenzzentrum Bankenarchitekturen im Informationszeitalter, St.Gallen, 2000

[Österle 1995]

Österle, H., Business Engineering, Prozess- und Systementwicklung, Band 1: Entwurfstechniken, Springer, Berlin et al., 1995

[Österle 1996]

Österle, H., Das Geschäftsmodell im Informationszeitalter, Rubrik Meinung/Dialog in:

Wirtschaftsinformatik 38, Nr. 4

[Österle 1999]

Österle, H., Vision des Business Engineering, Referat im MBE-Block 1, in: MBE-Lehrgangsunterlagen, Universität St.Gallen, Institut für Wirtschaftsinformatik, 1999

[Österle/Fleisch/Alt 2000]

Österle, H., Fleisch, E., Alt, R., Business Networking: Shaping Enterprise Relationships on the Internet, Springer, Berlin et al., 2000

[Quicken 2000]

o. V., Homepage des Finanzdienstleisters Quicken,

http://www.quicken.com/life_events/ (01.03.2000)

[Schierenbeck 1999]

Schierenbeck, H., Die Vertriebskanäle der Zukunft im Privatkundengeschäft, in: Basler Bankvereinigung (Hrsg.): Multi-Channel Distribution im Banking, Tagungsband zum 6. Basler Bankentag, Paul Haupt, Bern, 1999, S. 5-49

[Schlögel 1997]

Schlögel, M., Mehrkanalsysteme in der Distribution, Dissertation an der Universität St.Gallen, Dissertation, 1997 
[Schneider 1997]

Schneider, G., Multi-Channel-Management bei Banken, in: Bierbaum, D., Feinen, K. (Hrsg.): Bank- und Finanzwirtschaft: Strategien im Wandel, Gabler, Wiesbaden, 1997, S. 353-387

[Stäger 1999]

Stäger, C., Multi Channel Management: Mehrdimensionale Optimierung der Kanalbeziehungen zur nachhaltigen Steigerung der Profitabilität im Retail Banking, Paul Haupt, Bern, 1999

[Swisslife 2000]

o.V., Homepage des Finanzdienstleister Swiss Life,

http://www.swisslifeservices.ch/de/home/index.html (01.03.2000)

[Sydow 1992]

Sydow, J., Strategische Netzwerke - Evolution und Organisation, Gabler, Wiesbaden, 1992

[Timmers 1998]

Timmers, P.: Business Modells for Electronic Markets, in: Electronic Markets 8, 1998, Nr. 2

[Viscio/Pasternack 1996]

Viscio, A.J., Pasternack, B.A., 1996 Toward a New Business Model; Booz, Allen und Hamilton, http://www.strategy-business.com/research/96201 (06.06.2000)

[Zachman 1982]

Zachman, J.A., Business System Planning and Business Information Control Study: A Comparison, in: IBM Systems Journal 1/1982, S. 31-53 